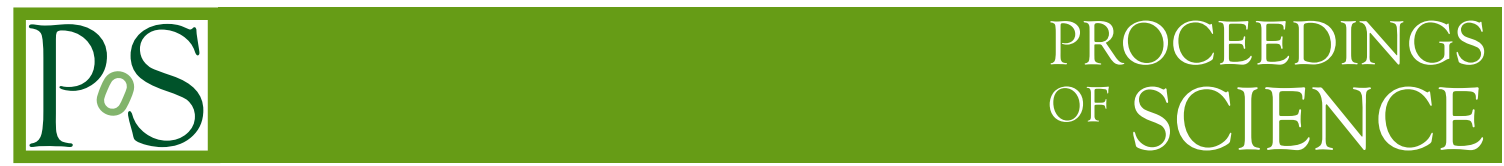

\title{
On the gluon propagator and Gribov noise in the Landau gauge gluodynamics
}

\author{
I.L. Bogolubsky* \\ JINR, Dubna \\ E-mail: bogolubsejinr.ru
}

I propose a way of a Gribov noise suppression when computing propagators in lattice approach and show the results for the gluon propagator which have been obtained in this way in the SU(2)

Landau gauge quenched QCD.

XXII International Baldin Seminar on High Energy Physics Problems

15-20 September, 2014

JINR, Dubna, Russia

${ }^{*}$ Speaker. 


\section{Introduction}

The infrared (IR) behavior of gauge-variant Green's functions (propagators) of Yang-Mills fields has been recently the subject of increased interest. Analytical investigations (in DysonSchwinger, DSE (see, e.g., [1] and refs. cited therein), and Functional Renormalization Group, FRG (see, e.g., [2]), approaches) have been stimulated, verified and completed with lattice studies (for the papers before 2006, see, e.g., [3]) of the gluon and the ghost propagators. Rather surprisingly it was found that the so-called 'conformal', or 'scaling', solution [4] is not the only possible solution, and another, 'regular', or 'decoupling', solution has been found $([5,6,7,8])$. When studying these problem researchers have to solve the Gribov copies problem [9] and to circumvent lattice artefacts due to the finite size, the finite spacing and hypercubic distortions. Due to above mentioned problems lattice studies of Yang-Mills propagators are very CPU-time and memory consuming and presently such simulations are done on modern supercomputers. Because of that improvement of simulation methods which leads to saving computer resources is of considerable importance for such studies.

\section{Gauge fixing and the Gribov copy problem}

We consider the Landau gauge Green functions (propagators) of $S U(N), N=2$, gluodynamics. We shall start with equilibrium ensembles of Yang-Mills (YM) lattice field configurations obtained as successive elements of Markov chains created in the course of Monte-Carlo generation when using the standard Wilson action. Lattice YM fields are defined by values of link variables $U_{x \mu} \in S U(N)$ which are transformed under gauge transformations $g_{x}$ as follows

$$
U_{x \mu} \stackrel{g}{\mapsto} U_{x \mu}^{g}=g_{x}^{\dagger} U_{x \mu} g_{x+\mu} ; \quad g_{x} \in S U(N) .
$$

Vector potentials of $S U(2)$ YM lattice fields are defined by the equation

$$
A_{x+\hat{\mu} / 2 ; \mu}=\frac{1}{2 i}\left(U_{x \mu}-U_{x \mu}^{\dagger}\right)
$$

we shall also use in what follows a shorter notation:

$$
\mathbf{A}_{x, \mu}=A_{x+\hat{\mu} / 2 ; \mu}
$$

The Landau gauge condition in the differential form reads

$$
\left(\partial_{\mu} A_{\mu}\right)(x)=\sum_{\mu=1}^{4}\left(A_{x+\hat{\mu} / 2 ; \mu}-A_{x-\hat{\mu} / 2 ; \mu}\right)=0 .
$$

It may be rewritten as an (integral) condition of an extremum of the gauge functional

$$
F_{U}(g)=\frac{1}{4 V_{4}} \sum_{x \mu} \frac{1}{N} \operatorname{Re} \operatorname{Tr} U_{x \mu}^{g}
$$

with respect to gauge transformations $g_{x}$, here $V_{4}=L^{4}$, where $L$ is the linear extension of the lattice. These (both differential and integral) conditions are satisfied on various configurations $\left(g_{x}\right)_{i}$, 
$i=1,2, \ldots N_{G r}$, which provide numerous solutions of gauge fixing problem ('Gribov ambiguity'). To avoid this ambiguity further specification of the Landau gauge fixing condition is required; we shall use for this purpose the requirement of attaining the global extremum of the gauge functional $F_{U}(g)$ on the configuration $\left(g_{x}\right)_{g l o b a l}$ (for the definition of $F_{U}(g)(2.5)$ it turns out to be its global maximum).

It was shown numerically that in lattice studies with Landau gauge-fixing for lattice extensions $L \geq 20$ the number of Gribov copies $N_{g r}$ is very large and thus an exact finding of the global extremum of $F_{U}(g)$ is hardly possible. Because of that a weaker choosing criterion is used in practical simulations, namely, a search for a Gribov copy $\left(g_{x}\right)_{0}$ having $F_{U}\left(g_{0}\right)$ as close as possible to $F_{U}\left(g_{\text {global }}\right)$. One of the most efficient numerical technique for getting closer to the global maximum of $F_{U}(g)$ is 'the simulated annealing' (SA) method [10] which for the first time was used in lattice studies in [11], and has been recently successfully applied to Landau gauge fixing of $S U(3)$ gluodynamics [7, 8]. The SA technique, sometimes called 'the stochastic optimization' method, realizes reasonably slow 'cooling' of the physical system defined by its 'action' $F_{U}(g)$ from an initial temperature $T_{0}$ to the final $T_{\text {fin }} \approx 0$, allowing at each temperature probabilistic 'tunneling through a potential barrier' which reduces probability of stucking the system in local minima. There exists the theorem stating that the SA process with infinitely slow 'cooling' guarantees arriving at the global extremum of the functional. However in practice computer resources are restricted and one can only hope to reach after sufficiently 'long' cooling a local extremum being 'rather close' to the global one.

In such situation the natural question arises: do numerical values of gauge-variant observables computed on different local extrema which are close to the (yet not found) global one differ considerably, or, in other words, is the so-called Gribov noise strong? A priori, one can think that the answer to this question is observable-dependent. Below we shall address this question when considering the ghost and the gluon propagators.

\section{The ghost propagator with SA}

Equation for the lattice ghost propagator are derived from lattice expression of the FaddeevPopov operator $M^{a b}$ :

$$
M_{x y}^{a b}=\sum_{\mu}\left\{\left(\bar{S}_{x \mu}^{a b}+\bar{S}_{x-\hat{\mu} ; \mu}^{a b}\right) \delta_{x ; y}-\left(\bar{S}_{x \mu}^{a b}-\bar{A}_{x \mu}^{a b}\right) \delta_{y ; x+\hat{\mu}}-\left(\bar{S}_{x-\hat{\mu} ; \mu}^{a b}+\bar{A}_{x-\hat{\mu} ; \mu}^{a b}\right) \delta_{y ; x-\hat{\mu}}\right\}
$$

where

$$
\bar{S}_{x \mu}^{a b}=\delta^{a b} \frac{1}{2} \operatorname{Tr} U_{x \mu} ; \quad \bar{A}_{x \mu}^{a b}=-\frac{1}{2} \varepsilon^{a b c} A_{x+\hat{\mu} / 2 ; \mu}^{c} .
$$

This is obtained as discretization of the continuum equation $M^{a b}=-\partial_{\mu} D_{\mu}^{a b}$, where $D_{\mu}^{a b}$ is the covariant derivative in the adjoint representation.

The ghost propagator $G^{a b}(x, y)$ is defined as $[12,13]$

$$
G^{a b}(x, y)=\delta^{a b} G(x-y) \equiv\left\langle\left(M^{-1}\right)_{x y}^{a b}[U]\right\rangle,
$$

where $M[U]$ is the Faddeev-Popov operator. The ghost propagator in the momentum space can be written as

$$
G(p)=\frac{1}{N V} \sum_{x, y} e^{-2 \pi i p \cdot(x-y)}\left\langle\left(M^{-1}\right)_{x y}^{a a}[U]\right\rangle,
$$




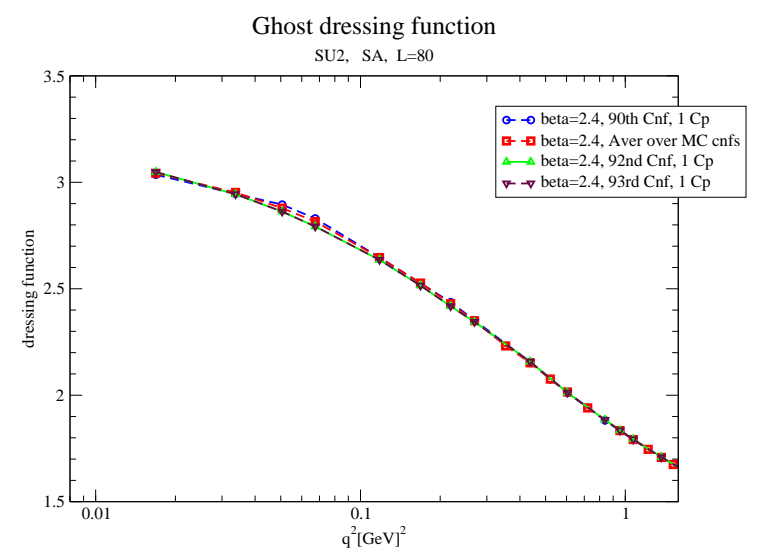

Figure 1: The ghost observable $J\left(q^{2}\right)$ for $\beta=2.4, L=80$ and various MC configurations compared with $J\left(q^{2}\right)$ averaged over MC configurations.

where $V=L^{4}$ is the lattice volume, and the coefficient $\frac{1}{N V}$ is taken for a full normalization, including the indicated color average over $a=1, \ldots, N$.

The ghost propagator has been subject of detailed lattice studies during two last decades $[13,14,15,16,17]$. For the present study we use the MC configurations produced for $\beta=2.4$ and $L=80$ in [18] and generate many 'good' Landau-gauge copies via gauge fixing with very slow SA cooling, so that all these Gribov copies have $F_{G}$ values very close to the global maximum value of $F_{G}$ (technically these essentially different copies have been found as results of the gauge fixing process starting from various random lattice $g(x)$ configurations). In Fig.1 we plot the ghost dressing function $J\left(q^{2}\right)=G\left(q^{2}\right) q^{2}$ computed on the first Gribov copy for different MC configurations. One can see that all the $J\left(q^{2}\right)$ curves are pretty smooth and rather close to the curve of the $J\left(q^{2}\right)$ averaged over $N_{g h}=26 \mathrm{MC}$ configurations for which the ghost propagator has been computed. This finding confirms the result obtained first in [7] that one can get reasonable approximation to the ghost $G\left(q^{2}\right)$ and $J\left(q^{2}\right)$ by computing the same curves from one (!) MC configuration with the only one 'good' Gribov copy calculated. This also means that Gribov noise for ghost observables is rather small and hence the conventional 'one-copy' algorithm of computation of $G\left(q^{2}\right)$ and $J\left(q^{2}\right)$ based on averaging of ghost observables over $N_{g h}$ MC configurations with one representative Gribov copy for each configuration is not far from being optimal. In the next section we shall see that such situation may change when considering another gauge-variant observable.

\section{Gribov noise for the gluon propagator with SA}

The lattice gluon propagators in the coordinate space is defined as

$$
D_{\mu v}^{a b}(x, y)=D_{\mu v}^{a b}(x-y)=\left\langle\mathbf{A}_{x, \mu}^{a} \mathbf{A}_{y, v}^{b}\right\rangle_{U} .
$$

However, of higher interest for physics is its analog in the momentum space

$$
D_{\mu \nu}^{a b}(q(p))=\frac{1}{V}\left\langle\sum_{x, y} \mathbf{A}_{x, \mu}^{a} \mathbf{A}_{y, v}^{b} e^{i p \cdot(x+\hat{\mu} / 2)} e^{-i p \cdot(y+\hat{v} / 2)}\right\rangle_{U},
$$




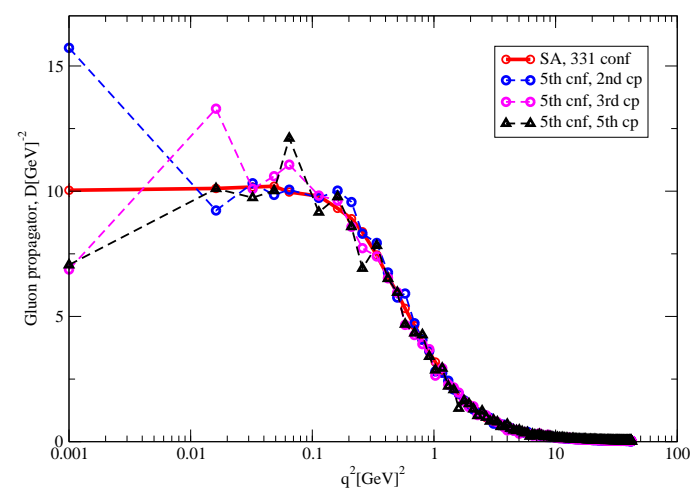

Figure 2: The gluon observable $D\left(q^{2}\right)$ for $\beta=2.4, L=80$, the 5th MC configuration and 3 Gribov copies compared with the $D\left(q^{2}\right)$ averaged over $N_{M C}$ configurations.

note that here summation over all relative distances $(x-y)$ available on the lattice is introduced to ensure translational invariance of the gluon propagator. We shall assume for the lattice propagator $D$ the same tensor structure which holds in the continuum theory,

$$
D_{\mu v}^{a b}(q)=\delta^{a b}\left(\delta^{\mu v}-\frac{q_{\mu} q_{v}}{q^{2}}\right) D\left(q^{2}\right)
$$

and extract from computer simulations the dependence $D\left(q^{2}\right)$ using an equation

$$
D\left(q^{2}\right)=\frac{1}{N^{2}-1} \sum_{a \mu} D_{\mu \mu}^{a a}(q)
$$

which follows from (4.2).

From previous lattice experience with gluon propagator it was known (see, e.g., [3]) that gluon curves $D\left(q^{2}\right)$ in the 'one-copy' approach may considerably differ for various configurations (cf. the ghost case in the above section). To get deeper insight into the gluon situation we have generated for several different $\mathrm{MC}$ configurations rather large number $N_{\text {copy }} \sim O(100)$ of 'good' Gribov copies and computed for them the gluon observable $D\left(q^{2}\right)$.

In Fig. 2 one can see that $D\left(q^{2}\right)$ curves for given MC configuration are strongly copy-dependent and are often 'saw-like'.

In Fig. 3 we plot more $D\left(q^{2}\right)$ curves for the 5th MC configuration and various 'good' Gribov copies. These curves are plotted together with the curve $D_{c p-a v}\left(q^{2}\right)$ obtained by averaging of the $D\left(q^{2}\right)$ over 77 Gribov copies found for the 5th MC configuration.

Analogous plot for the 10th MC configuration is presented in Fig.4, here the curve $D_{c p-a v}\left(q^{2}\right)$ is obtained by averaging of the $D\left(q^{2}\right)$ over 72 copies.

Now compare $D\left(q^{2}\right)$ results averaged over many Gribov copies of a single MC configuration with the results of the conventional averaging over $N_{M C}$ configurations with one Gribov copy chosen for each MC configuration ('the one-copy approach'). 


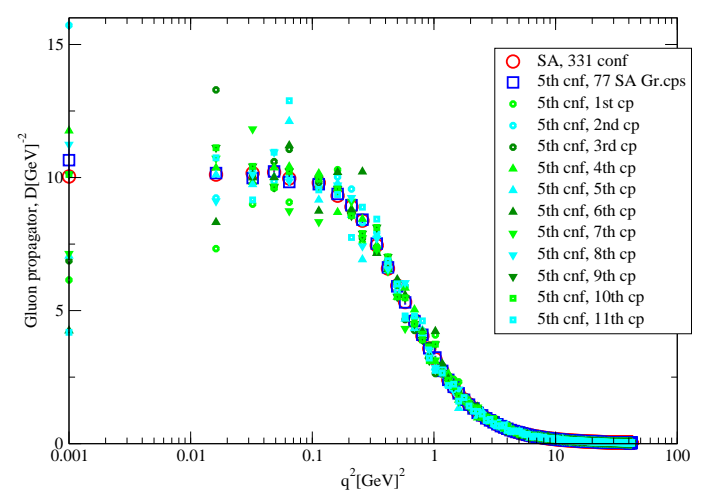

Figure 3: The gluon observable $D\left(q^{2}\right)$ for $\beta=2.4, L=80$, the 5th MC configuration and various Gribov copies compared with the $D\left(q^{2}\right)$ averaged over 77 SA Gribov copies.

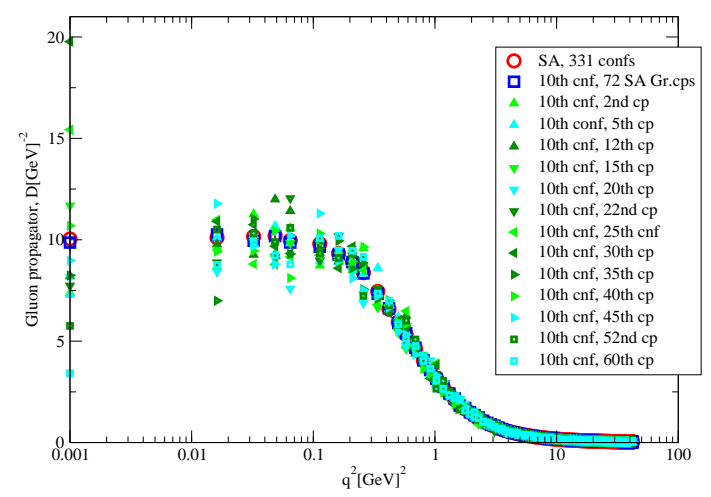

Figure 4: The gluon observable $D\left(q^{2}\right)$ for $\beta=2.4, L=80$, the 10th MC configuration and various Gribov copies compared with the $D\left(q^{2}\right)$ averaged over 72 SA Gribov copies.

One can see that averaging over various Gribov copies generated from one Yang-Mills MC configuration typically leads to the $D\left(q^{2}\right)$ dependence which is pretty close to the standard $D\left(q^{2}\right)$ propagator obtained by averaging over $N_{M C}$ MC configurations in the conventional 'one-copy' approach. Note that the $D\left(q^{2}\right)$ curves found in this way from various MC configurations also are of 'regular', or 'decoupling' type, thus showing qualitatively correct result. Moreover we can further average these new $D\left(q^{2}\right)$ results over relatively small number of MC configurations, for which 'the one configuration' $D\left(q^{2}\right)$ has been found by generating many 'good' Gribov copies; corresponding results will be published elsewhere. 


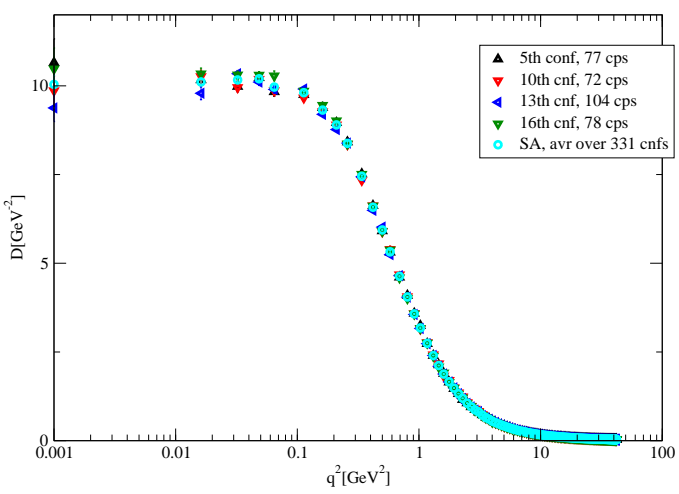

Figure 5: The gluon observable $D\left(q^{2}\right)$ for $\beta=2.4, L=80$, averaged over many Gribov copies for fixed MC configurations versus the gluon propagator $D\left(q^{2}\right)$ obtained in the 'one-copy approach' by averaging over $N_{M C}$ MC configurations.

\section{Conclusions}

We have found that when investigating the gluon propagator of lattice gluodynamics one is faced with the high level of Gribov noise, contrary to the case of the ghost propagator where Gribov noise is much weaker. We propose the way of overcoming this difficulty by means of averaging the gluon observable over many 'good' gauge copies found for the given Monte-Carlo configuration. Such averaging leads to qualitatively correct results for the gluon propagator $D\left(q^{2}\right)$, which means that from one MC configuration one can extract much more information on gaugevariant observables with high level of Gribov noise. This way of suppressing Gribov noise may be used for obtaining the gluon propagator (or another gauge-variant Green function for which the Gribov noise turns out to be strong) when only few MC configurations are available (for example, in the case of lattice simulations with dynamical fermions, when generating each MC configuration is very CPU-time consuming).

\section{Acknolegements}

The author is grateful to Prof. M. Müller-Preussker, E.-M. Ilgenfritz and A. Sternbeck for many useful discussions; possibility to use the computer program package for investigation of the Landau gauge propagators on parallel supercomputers which has been written by A. Sternbeck is thankfully acknoledged (but of course the author takes full responsibility for the results presented). Simulations have been done on the MVS100K supercomputer of the Joint Supercomputer Centre of RAS, Moscow.

\section{References}

[1] P. Boucaud, et al, JHEP, 06, 012 (2008); P. Boucaud, et al., JHEP, 06, 099 (2008). 
[2] C.S. Fisher, A. Maas, J.M. Pawlowski, Annals of Phys., 324, 2408 (2009).

[3] A. Sternbeck, arXiv:hep-lat/0609016.

[4] L. von Smekal, R. Alkofer, A. Hauk, Phys.Rev.Lett., 79, 3591 (1997); L. von Smekal, A. Hauk, R. Alkofer, Ann. Phys., 267, 1, (1998).

[5] A. Cucchieri, T. Mendes, PoS LAT2007, 297. (2007).

[6] A. Sternbeck, L. von Smekal, D.B. Leinweber, A.G. Williams, PoS LAT2007, 340 (2007).

[7] I.L. Bogolubsky, E.-M. Ilgenfritz, M. Müller-Preussker, A. Sternbeck, PoS LAT2007, 290 (2007).

[8] I.L. Bogolubsky, E.-M. Ilgenfritz, M. Müller-Preussker, A. Sternbeck, Phys.Lett., B676, 69 (2009).

[9] V.N. Gribov, Nucl. Phys., B139, 1 (1978).

[10] S. Kirkpatrick, C.D. Gelatt Jr., M.P. Vecchi, Science, 220, 671 (1983); V. Cherny, Journal of Optimization Theory and Applications, 45, 41 (1985).

[11] G.S. Bali, V. Bornyakov, M. Müller-Preussker, F. Pahl, Nucl. Phys. Proc. Suppl., 42, 852 (1995).

[12] D. Zwanziger, Nucl. Phys., B412, 657 (1994).

[13] H. Suman and K. Schilling, Phys. Lett., B373, 314 (1996).

[14] A. Cucchieri, Nucl. Phys., B508, 353 (1997).

[15] H. Nakajima and S. Furui, Nucl. Phys., B (Proc. Suppl.) 73, 865 (1999); Nucl. Phys., B (Proc. Suppl.) 83, 521 (2000); Nucl. Phys., A680, 151 (2000); H. Nakajima, S. Furui and A. Yamaguchi, Nucl. Phys., B (Proc. Suppl.) 94, 558 (2001); H. Nakajima and S. Furui, Nucl. Phys., B (Proc. Suppl.) 119, 730 (2003).

[16] J.C.R. Bloch, A. Cucchieri, K. Langfeld and T. Mendez, Nucl. Phys., B (Proc. Suppl.) 119, 736 (2003);

K. Langfeld, J.C.R. Bloch, J. Gattnar, H. Reinhardt, A. Cucchieri and T. Mendes, talk given at 5th International Conference on Quark Confinement and the Hadron Spectrum, Gargnano, Brescia, Italy, 10-14 Sep 2002, edited by N. Brambilla, G. M. Prosperi; River Edge, N.J., World Scientific (2003), pp. 297-299; arXiv: hep-th/0209173.

[17] T. Bakeev, E.-M. Ilgenfritz, M. Müller-Preussker and V.K. Mitrjushkin, Phys. Rev., D69, 074507 (2004).

[18] I.L. Bogolubsky, E.-M. Ilgenfritz, M. Müller-Preussker, A. Sternbeck, PoS Confinement X, 285 (2012). 